\title{
Twin Paradox and Space Topology
}

\author{
Jean-Philippe Uzan ${ }^{1,2}$, Jean-Pierre Luminet ${ }^{3}$, Roland Lehoucq ${ }^{4}$ and Patrick Peter ${ }^{3,5}$ \\ (1) Laboratoire de Physique Théorique, CNRS-UMR 8627, Bât. 210, Université Paris XI, F-91405 Orsay cedex (France) \\ (2) Département de Physique Théorique, Université de Genève, 24 quai E. Ansermet, CH-1211 Genève 4 (Switzerland) \\ (3) Département d'Astrophysique Relativiste et de Cosmologie, Observatoire de Paris, CNRS-UMR 8629, \\ F-92195 Meudon cedex (France) \\ (4) CE-Saclay, DSM/DAPNIA/Service d'Astrophysique, F-91191 Gif sur Yvette cedex (France). \\ (4) Institut d'Astrophysique de Paris, 98 bis, boulevard Arago, 75005 Paris (France)
}

(February 2, 2008)

\begin{abstract}
If space is compact, then a traveller twin can leave Earth, travel back home without changing direction and find her sedentary twin older than herself. We show that the asymmetry between their spacetime trajectories lies in a topological invariant of their spatial geodesics, namely the homotopy class. This illustrates how the spacetime symmetry invariance group, although valid locally, is broken down globally as soon as some points of space are identified. As a consequence, any non-trivial space topology defines preferred inertial frames along which the proper time is longer than along any other one.
\end{abstract}

Preprint numbers: LPT-ORSAY 00/45, UGVA-DPT 00/04-1080

\section{INTRODUCTION}

The twin paradox is the best known thought experiment of special relativity, whose resolution provides interesting insights on the structure of spacetime and on the applicability of the Lorentz transformations. In its seminal paper on special relativity, Einstein [1] pointed out the problem of clocks synchronization between two inertial frames with relative velocity $v$. Later on, Langevin [2] picturesquely formulated the problem by taking the example of twins aging differently according to their respective worldlines. The keypoint for understanding the paradox is the asymmetry between the spacetime trajectories of the "sedentary twin" and of the "traveller twin". The subject has been widely studied for pedagogical purpose 3., 1], the role of acceleration was examined in details [5 8] and a full general relativistic treatment was given [9].

Although counter-intuitive, the twin paradox is clearly not a logical contradiction, it merely illustrates the elasticity of time in relativistic mechanics. The experiment was actually performed in 1971 with twin atomic clocks initially synchronized, one of them being kept at rest on Earth and the other one being embarked on a commercial flight: the time shifts perfectly agreed with the fully relativistic calculations [10]

An interesting "revisited" paradox was formulated 111,12 in the framework of a closed space (due to curvature or to topology). In such a case, the twins can meet again without none of them being accelerated, yet they aged differently. Both an algebraic and a geometric solution were given [13].

Our present goal is to extend such explanations by adding a topological characterization of reference frames, which allows us to solve the twin paradox whatever the global shape of space may be. We first briefly recall, in $\S$ III. the classical twin paradox and its standard resolu- tion, in $\S$ III we investigate the case of a spacetime with compact spatial sections and in $\S$ IV, we show that the root explanation of the twin paradox lies in the global breakdown of the spacetime symmetry group by a nontrivial topology.

\section{THE STANDARD TWIN PARADOX}

Let observers 1 and 2 be attached to inertial frames with relative velocity $v$. 1 is supposed to be at rest and to experience no acceleration. At time $t=0$, the observers synchronise their clock (thus they can be called "twins"). Then twin 2 travels away at velocity $+v$ with respect to 1 and comes back with velocity $-v$. According to special relativity, the travel time, $\Delta \tau_{2}$, measured by 2 (its proper time) is related to the proper time measured by $1, \Delta \tau_{1}$, by

$$
\Delta \tau_{2}=\sqrt{1-v^{2}} \Delta \tau_{1} .
$$

A paradox arises if one considers that the situation is perfectly symmetrical about 1 and 2 , since 2 sees 1 travelling away with velocity $-v$ and coming back with velocity $+v$. If this was correct, one could reverse the reasoning to deduce that 1 should be younger than 2 , with

$$
\Delta \tau_{1}=\sqrt{1-v^{2}} \Delta \tau_{2},
$$

and an obvious contradiction would arise.

Indeed, the previous argument holds whenever 2 is not accelerating. As first explained by Paul Langevin [2], among all the worldlines that connect two spacetime events (such as the departure and return of 2), the one which has the longest proper time is the unaccelerated one, i.e. the reference frame $\mathbf{K}$ of 1 . The traveller twin 2 cannot avoid accelerating and decelerating to make its return journey; then he had to jump from an inertial frame 
$\mathbf{K}^{\prime}$ moving relatively to $\mathbf{K}$ with velocity $v$ to another inertial frame $\mathbf{K}^{\prime \prime}$ moving with velocity $-v$ with respect to $\mathbf{K}$. Hence the situation is not symmetrical about the twins: a kink (infinite acceleration) in the middle of the path of twin 2 explains the difference, and there is no contradiction in the fact that the sedentary twin 1 will definitely be older than the traveller twin 2 .

The same result holds in the framework of general relativity, dealing with a more realistic situation including accelerations, gravitational fields and curved spacetime (so that the kink is smoothed out): in order to achieve its journey, the traveller 2 necessarily experiences a finite and variable acceleration; thus her reference frame is not equivalent to that of 1 .

Such explanations, as rephrased by Bondi 14, are equivalent to say that there is only one way of getting from the first meeting point to the second without acceleration.

However, acceleration is not the only and essential point of the twin paradox, as shown by the example of non-accelerated twins in a closed space, in which there are several ways to go from the first meeting point to the second one without accelerating 11$][13$. The key explanation of the twin paradox is now "some kind" of asymmetry between the spacetime paths joining two events. We investigate below the nature of such an asymmetry when space topology is not trivial (i.e. simplyconnected)

\section{TWINS IN A COMPACT SPACE}

In a spacetime which has at least one compact space dimension, one can actually start from one point, travel along several straight geodesics and come back to the same spatial position without accelerating nor decelerating. Einstein's relativity theory determines the local properties of the spacetime $\mathcal{M}$ (its metrics), but gives little information about its global properties (its topology) 15, 16, so that special relativity (in the absence of gravitational fields) or general relativity (involving gravitational fields in curved spacetimes) well describe the local physics. For instance, the Minkowski spacetime $(\mathcal{M}, \eta)$ used in special relativity is a manifold $\mathbf{R}^{4}$ with a flat Lorentzian metric $\eta=\operatorname{diag}(-1,1,1,1)$ and Euclidean space sections $\Sigma$. One can obain spacetimes locally identical to $(\mathcal{M}, \eta)$ but with different large scale properties by identifying points in $\mathcal{M}$ under a group of transformations, called holonomies, which preserve the metric (thus holonomies are isometries). For instance, identifying $\left(x_{0}, x_{1}, x_{2}, x_{4}\right)$ with $\left(x_{0}+L, x_{1}, x_{2}, x_{3}\right)$ changes the topology from $\mathbf{R}^{4}$ to a cylinder $\mathbf{S}^{1} \times \mathbf{R}^{3}$ and introduces closed timelike lines. However, if causality is believed to hold in the sense that no effect can preceed its cause, such an identification is prohibited, and the study of spacetime topology is restricted so as to exclude closed timelike curves [15]. This is achieved if spacetime can be decomposed as the direct product

$$
\mathcal{M}=\mathbf{R} \times \Sigma
$$

where the real axis $\mathbf{R}$ refers to the time direction and $\Sigma$ to the three dimensional spatial sections. Now the topology of spacetime amounts to the study of the various shapes of the spatial sections $\Sigma$.

The topology of a three-dimensional Riemannian space $\Sigma$ can be described in full generality by a fundamental polyhedron $\mathcal{P}$ and a holonomy group $\Gamma$ whose elements $g$ identify the faces of the polyhedron by pairs (see e.g. 16] for a general discussion of the topological properties of spacetimes). It follows that $\Sigma$ can be written as

$$
\Sigma=X / \Gamma
$$

where $X$ is the universal covering space 16] (simplyconnected and three dimensional) and the quotient refers to the equivalence relation ' $\equiv$ ' defined as

$$
\forall \mathbf{x}, \mathbf{y} \in X \quad \mathbf{x} \equiv \mathbf{y} \Longleftrightarrow(\exists g \in \Gamma \quad \mid \quad \mathbf{x}=g \mathbf{y}) .
$$

If $\Gamma$ reduces to the identity, space is simply-connected, in the sense that two points of space are connected by only one geodesic. As soon as there are nontrivial holonomies which identify points, space is multiconnected and several geodesics connect two any distinct points. In a cosmological context, multi-connected universe models lead to the existence of ghost images in the observable universe when one topological length is shorter than the horizon size. Many methods aimed to detect the cosmic topology have been proposed [16,17, so far with no definite answer coming from observational data.

Returning to Minkowski space for the sake of clarity, the holonomies of $\Gamma$ that preserve the flatness of the space sections $\Sigma$ are the identity, the translations, the reflexions and the helicoidal displacements. The group classification leads to 18 Euclidean spaceforms with different topologies, all of them having as universal covering space $X$ the simply-connected, infinite Euclidean space $\mathbf{R}^{3}$. Six of them are compact (i.e. of finite volume) and orientable. Their fundamental polyhedron can be a parallelepiped or a hexagonal prism. For the sake of visualization, in the following we shall develop our reasoning in flat spacetimes with $1+2$ dimensions only, i.e. whose spatial sections $\Sigma$ are just surfaces. In such a case there are 5 Euclidean topologies: the cylinder, the Möbius strip, the flat torus, the Klein bottle and the Euclidean plane [16]. For a pedagogical purpose, we select the case where space has a torus-like topology (see figure 11). However we emphasize that our conclusions will remain valid in $(1+3)$ dimensions, whatever the topology and the (constant) space curvature may be. 


\begin{tabular}{|l|l|l|l|l|}
\hline Name & $\begin{array}{c}\text { FD and } \\
\text { identifi- } \\
\text { cations }\end{array}$ & Shape & Closed & Orientable \\
\hline cylinder & NO & YES \\
\hline $\begin{array}{c}\text { Möbius } \\
\text { strip }\end{array}$ & Nein \\
\hline torus & & Nottle \\
\hline
\end{tabular}

FIG. 1. The four multi-connected topologies of the two dimensional Euclidean plane. They are constructed from a parallelogram or an infinite band (fundamental domain FD), by identification of edges according to the allowable holonomies. We indicate as well their compactness and orientability properties (from [14]).

Since a characteristics of multi-connected spaces is to provide several geodesics between two any points, we shall extend the concept of "twins" to more than two and consider an ensemble of "twins" (strictly speaking, initially synchronized clocks) labeled as 1,2,3 and 4 (see figure 2 [left]). The twin 1 stays at home at point $O$ and her worldline can be identified with the time axis. The twin 2 leaves $O$ at $t=0$, travels away and then turns back to meet twin 1 in $P$. The twins 3 and 4 leave $O$ in two different directions along non-accelerated worldlines and travel away from 1 ; they respectively reach $P^{\prime}$ and $P^{\prime \prime}$, where they meet twin 1 . Due to warped space, twins 3 and 4 managed to come back without changing their direction and inertial frame. Now, one wants to compare the ages within the various pairs of twins when they meet again. Whereas twin 2 undergone the standard paradox, there seems to be a real paradox with twins 1, 3 and 4, who all followed strictly inertial frames.

In [13] it was shown that, in the case of a cylinder, the sedentary twin 1 is always older than any traveller because their states of motions, although non-accelerated, are not symmetrical. Which kind of asymmetry is to be considered? As we emphasize below, acceleration is a local concept, and the only explanation lies in a global breakdown of symmetry due to a non-trivial topology.

Let us consider the projection of the worldlines onto a constant time hypersurface $\Sigma$, assumed to be a $2 \mathrm{D}$ flat torus (see figure 2 [right]). Each projection is a loop $\gamma\left(u, \mathbf{x}_{0}\right)$ at $\mathbf{x}_{0}$ which can be parametrised by $u \in[0,1[$

\footnotetext{
${ }^{*}$ Under very special circumstances, twins 3 and 4 could also meet altogether with twins 1 and 2 at the same point of spacetime!
}

if $\gamma(0)=\gamma(1)=\mathbf{x}_{0}$, where $\mathbf{x}_{0}$ is a point of $\Sigma$. Two loops at $\mathbf{x}_{0}, \gamma$ and $\delta$, are said to be homotopic $(\gamma \sim \delta)$ if they can be continuously deformed into one another, i.e. if there exists a continuous map (called homotopy) $F:[0,1[\times[0,1[\rightarrow X$ such that

$$
\begin{gathered}
\forall u \in[0,1[, \quad F(s, 0)=\gamma(u), F(u, 1)=\delta(u) \\
\forall v \in\left[0,1\left[, \quad F(0, v)=F(1, v)=\mathbf{x}_{0} .\right.\right.
\end{gathered}
$$

The equivalence class of homotopic loops is denoted by $[\gamma]$. We denote $\gamma_{i}$ the loop corresponding to the projection of the trajectory of twin $i$ in $\Sigma$.

In our example (see figure 2), the twins 1 and 2 have homotopic trajectories: since 2 does not "go around" the universe, the loop $\gamma_{2}$ can be continuously contracted into the null loop $\gamma_{1}=\{0\}$, so

$$
\gamma_{1} \sim \gamma_{2} \sim\{0\}
$$

However, among these two homotopic loops, only one corresponds to an inertial observer going from $O$ to $P$ : that of twin 1, which is thus older than twin 2, as expected in the standard paradox.

Now, the twins 3 and 4 respectively go once around the hole and around the handle of the torus. From a topological point of view, their paths can be characterized by a so-called winding index. In a cylinder, the winding index is just an integer which counts the number of times a loop rolls around the surface. In the case of a 2 -torus, the winding index is a couple $(m, n)$ of integers where $m$ and $n$ respectively count the numbers of times the loop goes around the hole and the handle. In our example, twins 1 and 2 have the same winding index $(0,0)$, whereas twins 3 and 4 have winding indexes respectively equal to $(1,0)$ and $(0,1)$. The winding index is a topological invariant for each traveller: neither change of coordinates or reference frame (which ought to be continuous) can change its value.

To summarize, we have the two situations:

1. Two twins have the same winding index (twins 1 and 2 in our example), because their loops belong to the same homotopy class. Nevertheless only one (twin 1) can go from the first meeting point to the second one without changing inertial frame. The situation is not symmetrical about 1 and 2 due to local acceleration, and 1 is older than 2 .

2. Several twins (1, 3 and 4 in our example) can go from the first meeting point to a second one at a constant speed, but travel along paths with different winding indexes. Their situations are not symmetrical because their loops belong to different homotopy classes:

$$
\gamma_{1} \not \gamma_{3} \not \gamma_{4}
$$

Twin 1 is older than twins 3 and 4 because her path has a zero winding index. 

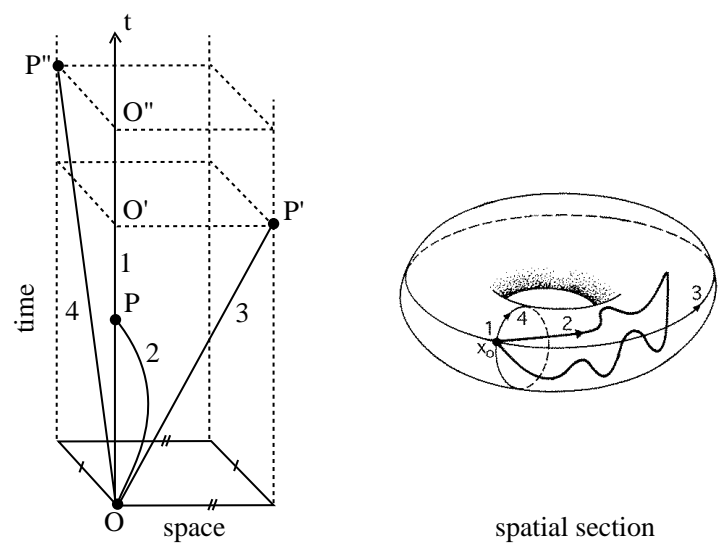

FIG. 2. Different twins in a $(1+2)$ spacetime with toroidal spatial sections. All of them leave 0 at the same time. While 1 remains at home, 2 [dash-dotted line] goes away and then comes back to meet 1 in $P$ (it corresponds to the standard case), 3 [solid line] goes around the universe in a given direction from $O$ to $P^{\prime} \equiv O^{\prime}$ and 4 [dashed line] goes around the universe along another direction from $O$ to $P^{\prime \prime} \equiv O^{\prime \prime}$. On the left plot, we used the fundamental domain representation (in which the 2-torus corresponds to a rectangle whose edges / and // are respectively identified; see figure 1). On the right plot, we depict the projection of their trajectory on a constant time slice. The spacetime points $O, O^{\prime}, O^{\prime \prime}, P, P^{\prime}$ and $P^{\prime \prime}$ of $\mathcal{M}$ are projected onto the same base point " 1 " in $\Sigma$.

In order to solve exhaustively the twin paradox in a multi-connected space, one would like not only to compare separately the ages of the travellers with the age of the sedentary twin, but also to compare the ages of the various travellers when they respectively meet each other. It is clear that the only knowledge of the winding index of their loops does not allow us, in general, to compare their various proper time lapses. The only exception is that of the cylinder, where a larger winding number always corresponds to a shorter proper time lapse. But for a torus of unequal lengths, for instance when the diameter of the hole is much larger than the diameter of the handle, a traveller may go straight around the handle many times with a winding index $(0, n)$, and yet be older than the traveller which goes straight only once around the hole with a winding index $(1,0)$. The situation is still more striking with a double torus, indeed a hyperbolic surface instead of an Euclidean one (see e.g. [16]). The winding indexes become quadruplets of integers and, as for the simple torus, they cannot be compared to answer the question on the ages of the travellers. As we shall see below, this problem can be solved only by using an additional metric information.

\section{LANGEVIN AND POINCARÉ}

We have found a topological invariant attached to each twin's worldline which accounts for the asymmetry between their various inertial reference frames. Why is it so? In special relativity theory, two reference frames are equivalent if there is a Lorentz transformation from one frame of space-time coordinates to another system. The set of all Lorentz transformations is called the Poincaré group - a ten dimensional group which combines translations and homogeneous Lorentz transformations called "boosts".

The non equivalence between the inertial frames is due to the fact that space topology breaks globally the Poincaré group. Indeed, cutting and pasting to compactify the spatial sections defines (i) particular directions, so that space, even if locally isotropic, is no more invariant under rotations, and (ii) a particular time: the one measured by an observer whose 4 -velocity is perpendicular to $\Sigma$ (the twin 1 in our example). Let us call $t$ the proper time of such an observer; then the spacetime coordinates $p$ of any point $P \in \mathcal{M}$ can be decomposed in the inertial frame $\mathbf{K}$ as $p=(t, \mathbf{x})$, and the choice of a topology reduces to the choice of the identifications

$$
p=(t, \mathbf{x}) \equiv(t, g \mathbf{x})=g(p), \quad g \in \Gamma .
$$

In the inertial frame $\mathbf{K}^{\prime}$ of the traveller, the coordinates of $P$ are given by a Lorentz transformation $\mathcal{L}(p)=\left(t^{\prime}, \mathbf{x}^{\prime}\right)$ with

$$
t^{\prime}=\gamma(t-\mathbf{v} \cdot \mathbf{x}), \quad \mathbf{x}^{\prime}=\gamma(\mathbf{x}-\mathbf{v} t)
$$

where $\gamma \equiv\left(1-v^{2}\right)^{-1 / 2}$. It is clear from (6) and (7) that

$$
\forall g \nsim I_{d}, \forall p, \quad g \circ \mathcal{L}(p) \neq \mathcal{L} \circ g(p) .
$$

The identification (6) particularises a given foliation and spatial sections, leading to the existence of an absolute rest frame (the one of zero homotopy class). For any other observers, these identifications are relations between events at different times (and thus in different spatial sections) and not a relation between points in a given spatial section. As pointed in [12], the observers 3 and 4 will find that their constant time hypersurfaces do not match in the universal covering space and that there are points on these surfaces of simultaneity which are connected by timelike curves. Moreover, the only holonomy $g$ such that $g \circ \mathcal{L}(p)=\mathcal{L} \circ g(p)$ for all $p$ is the identity $g=I_{d}$, thus the holonomy group reduces to $\Gamma=\left\{I_{d}\right\}$ and $\Sigma$ reduces to $X$. We deduce that the only topology compatible with the Poincaré group is the trivial topology. In other words, the only flat spaceform invariant under the full Poincaré group is the original simply-connected Minkowski spacetime, and any additional discrete identification group is incompatible with the Lorentz transformations.

In conclusion, the oldest twin will always be the one of homotopy class $\{0\}$, and between two twins of same homotopy class, the oldest one will be the one who does not undergo any acceleration. We can rephrase Bondi's formulation of the solution by saying that "there is only one way of a given homotopy class of getting from the first meeting point to the second without acceleration". 
This generalises the previous works [11 13 ] by adding topological considerations which are more general and hold whatever the shape of space is. As concluded in [12], "it is not sufficient that [the] motion [of the twin] is symmetrical in terms of acceleration felt and so on; it must also be symmetrical in terms of the way that their worldlines are embedded into the spacetime"; this latter symmetry is the one we have exhibited and which is encoded in the homotopy class.

As mentioned in the previous section, the homotopy classes only tell us which twin is aging the fastest: the one who follows a straight loop homotopic to $\{0\}$. It does not provide a classification of the ages (i.e. proper time lengths) along all the straight loops. To do this, some additional information is necessary, such as the various identification lengths. Indeed there exists a simple criterion which works in all cases: a shorter spatial length in the universal covering space will always correspond to a longest proper time. To fully solve the question, it is therefore sufficient to draw the universal covering space as tessellated by the fundamental domains, and to measure the lengths of the various straight paths joining the twin 1 position in the fundamental domain to its ghost positions in the adjacent domains (see figure 3). As usual in topology, all reasonings involving metrical measurements can be solved in the simply-connected universal covering space.

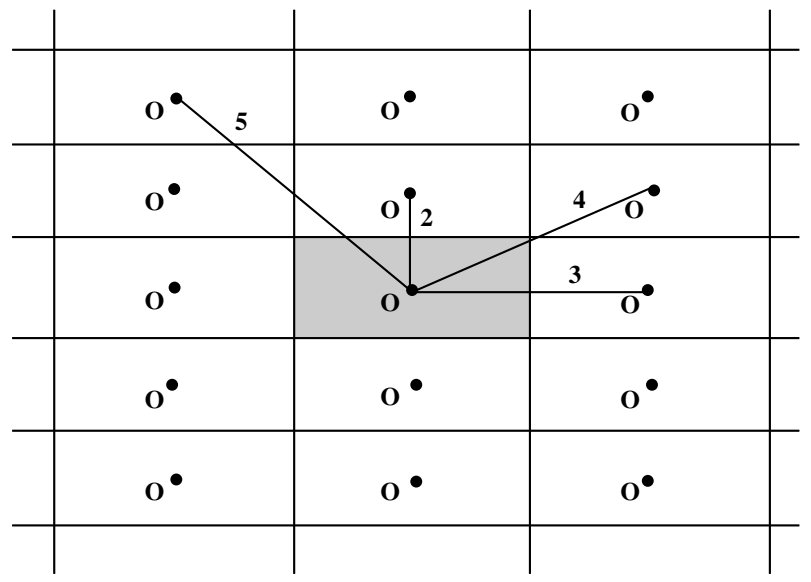

FIG. 3. Straight paths in the universal covering space of a $(2+1)$-spacetime with flat, torus-like spatial sections. Paths $2,3,4,5$ are straight loops with respective winding indexes $(0,1),(1,0,(1,1),(1,2)$, allowing the traveller twins to leave and meet again the sedentary twin $O$ without accelerating. The inertial worldlines are clearly not equivalent: the longest the spatial length in the universal covering space, the shortest the proper time travel in spacetime.

In the framework of general relativity, general solutions of Einstein's field equations are curved spacetimes admitting no particular symmetry. However, all the exact known solutions admit symmetry groups (although less rich than the Poincaré group). For instance, the usual "big bang" cosmological models - described by the Friedmann-Lemaître solutions - are assumed to be globally homogeneous and isotropic. From a geometrical point of view, this means that spacelike slices have constant curvature and that space is spherically symmetric about each point. In the language of group theory, the spacetime is invariant under a six-dimensional isometry group. The universal covering spaces of constant curvature are $\mathbf{R}^{3}, \mathbf{S}^{3}$ or $\mathbf{H}^{3}$ according to the zero, positive or negative value of the curvature. Any identification of points in these simply-connected spaces via a holonomy group lowers the dimension of their isometry group If; it preserves the three-dimensional homogeneity group (spacelike slices have still constant curvature), but it breaks down globally the isotropy group (at a given point there are a discrete set of preferred directions along which the universe does not look the same).

Thus in Friedmann-Lemaître universes, (i) the expansion of the universe and (ii) the existence of a non-trivial topology for the constant time hypersurfaces both break the Poincare invariance and single out the same "privileged" inertial observer who will age more quickly than any other twin: the one comoving with the cosmic fluid although aging more quickly than all her travelling sisters may be not a real privilege!

[1] A. Einstein, "Zur Elektrodynamik bewegter Körper", Ann. Phys. (Leipzig) 17, 891 (1905).

[2] P. Langevin, "L'évolution de l'espace et du temps", Scientia X, 31 (1911).

[3] R.H. Romer, "Twin paradox in special relativity", Am. J. Phys. 27, 131-135 (1959).

[4] E. F. Taylor and J.A. Wheeler, Spacetime Physics, Second Edition, Freeman, 1992.

[5] W.G. Unruh, "Parallax distance, time, and the twin paradox", Am. J. Phys. 49 (6), 589-592 (1981).

[6] H. Nikolić, "Relativistic contraction of an accelerated rod", Am. J. Phys. 67 (11), 1007-1012 (1999).

[7] R.H. Good, "Uniformly accelerated reference frame and twin paradox", Am. J. Phys. 50, 232-238 (1982).

[8] S.P. Boughn, "The case of the identically accelerated twins", Am. J. Phys. 57 (9), 791-793 (1989).

[9] R. Perrin, "Twin paradox: a complete treatment from the point of view of each twin", Am. J. Phys. 44, 317319 (1970).

[10] J.C. Hafele and R.E. Keating, "Around-the-world atomic clocks: Observed relativistic time gains", Science, 177, 168-170 (1972).

[11] C.H. Brans and D.R. Stewart, "Unaccelerated returning

\footnotetext{
${ }^{\dagger}$ There is one exception: the projective space, obtained by identifying the antipodal points of $\mathbf{S}^{3}$
} 
twin paradox in flat spacetime", Phys. Rev. D8, 16621666 (1973).

[12] R.J. Low, "An acceleration free version of the clock paradox", Eur. J. Phys. 11, 25-27 (1990).

[13] T. Dray, "The twin paradox revisited", Am. J. Phys. 58 (9), 822-825 (1990).

[14] H. Bondi, Relativity and common sense, Doubleday and Company Inc., N.Y. (1964).

[15] R. Geroch, "Space-time structure from a global viewpoint", in General relativity and cosmology, proceedings of course XLVII of the international course of physics "Enrico Fermi", Ed. R.K. Sachs, Academic Press (New York, 1971).

[16] M. Lachièze-Rey, J.-P. Luminet, "Cosmic topology", Phys. Rep. 254, 135 (1995).

[17] W.P. Thurston and J. Weeks, "The Mathematics of Three-Dimensional Manifolds", Sci. Am., 251, 108-113 (July 1984); J-P. Luminet, G.D. Starkman and J.R. Weeks, "Is space finite?", Sci. Am., 280, 90-97 (april 1999); J-P. Uzan, R. Lehoucq and J-P. Luminet, "New developments in the search for the topology of the universe", Proc. of the XIX ${ }^{\text {th }}$ Texas meeting, Paris 14-18 december 1998, Eds. E. Aubourg, T. Montmerle, J. Paul and P. Peter, article $n^{\circ} 04 / 25$. 